\title{
A New Approach to IRMPD Using Selective Ion Dissociation in a Quadrupole Ion Trap
}

\author{
G. Asher Newsome, Gary L. Glish
}

Department of Chemistry, University of North Carolina at Chapel Hill, Chapel Hill, NC 27599-3290, USA

\begin{abstract}
Infrared multiphoton photodissociation (IRMPD) in a quadrupole ion trap is not selective for a parent ion. Product ions are decreased in abundance by continuous sequential dissociation and may be lost below the low mass cut-off. The IRMPD process is made selective by resonantly exciting trapped ions into an axially offset laser path. Product ions form and collisionally relax out of the laser path to accumulate in the center of the trap. The technique, termed selective broadband (SB) IRMPD, limits sequential dissociation to preserve first generation product ion abundance. The abundances of larger product ions are maximized by completely dissociating the parent ion, but continuous sequential dissociation does not form small product ions below the low mass cut-off associated with conventional IRMPD. Smaller product ions are further increased in abundance in another tandem mass spectrum by performing sequential stages of SB-IRMPD, adjusting the trapping if amplitude to dissociate larger product ions at the same $q_{z}$ range. Thermal assistance is used to perform SB-IRMPD at higher bath gas pressures for increased sensitivity.
\end{abstract}

Key words: Infrared multiphoton phodissociation, Collision-induced dissociation, Tandem massspectrometry, Quadrupole ion trap, Peptide

\section{Introduction}

Q uadrupole ion trap mass spectrometers can perform multiple stages of tandem mass spectrometry on trapped ions. Collision-induced dissociation (CID) is the most common method for dissociating trapped ions, but infrared multiphoton photodissociation (IRMPD) is a useful and increasingly popular alternative [1-7]. In conventional CID only one mass-to-charge ion is selected for excitation, limiting sequential dissociation to form low mass product ions. CID requires a low mass cut-off (LMCO) at about onethird to one-fourth the mass-to-charge of the parent ion. IRMPD has a smaller LMCO than CID and is used to observe a broader mass-to-charge range of product ions [2,

Electronic supplementary material The online version of this article (doi:10.1007/s13361-010-0039-y) contains supplementary material, which is available to authorized users.

Correspondence to: Gary L.Glish; e-mail: glish@unc.edu
3]. IRMPD produces high parent ion fragmentation efficiency $[8,9]$,

$$
E_{F}=\frac{\sum_{i} F_{i}}{\sum_{i} F_{i}+P} \times 100 \%
$$

where $E_{F}$ is fragmentation efficiency, $F_{i}$ is the abundance of each product ion, and $\mathrm{P}$ is the abundance of the parent ion remaining after irradiation. By irradiating all parent and product ions simultaneously, continuous sequential dissociation generates immonium ions and other identifiable low mass $(<m / z$ 200) product ions from peptides and proteins that can contribute useful information to tandem mass spectra. By trapping more product ions at a lower LMCO, IRMPD also can give a higher MS/MS efficiency $[8,9]$ than CID,

$$
E_{M S / M S}=\frac{\sum_{i} F_{i}}{P_{0}} \times 100 \%
$$

Received: 29 September 2010

Revised: 8 December 2010

Accepted: 8 December 2010

Published online: 15 January 2011 
where $\mathrm{E}_{\mathrm{MS} / \mathrm{MS}}$ is MS/MS efficiency, $\mathrm{F}_{\mathrm{i}}$ is the abundance of each product ion, and $\mathrm{P}_{0}$ is the initial abundance of the parent ion before irradiation. A limitation to the use of IRMPD in a quadrupole ion trap compared with CID has been minimal fragmentation efficiency at $1.0 \times 10^{-3}$ Torr, the bath gas pressure necessary for optimal sensitivity [10]. Bath gas collisions reduce the kinetic energy of ions during injection to increase trapping efficiency [11] but also collisionally cool the internal energy of trapped ions [12]. IRMPD is typically performed at $1 \times 10^{-5}$ to $3 \times 10^{-4}$ Torr to decrease collisional cooling of parent ion internal energy [27, 12]. A variety of methods have been developed to increase fragmentation efficiency with supplemental ion activation and/or by decreasing the rate of collisional cooling $[1,2,10,13,14]$.

The lack of selectivity of IRMPD performed at any bath gas pressure, with or without modifications to improve fragmentation efficiency, reduces the possible sensitivity of the technique. The continuous irradiation of parent and product ions in IRMPD makes it difficult to control the extent of sequential dissociation [3]. More product ions are observed if $100 \%$ fragmentation efficiency of the parent ion is achieved, but each product ion abundance is decreased. Continuous sequential dissociation, especially to observe lower mass product ions, may produce ions that fall below the LMCO, which lowers the MS/MS efficiency. The decreased product ion abundances and ion losses below the LMCO in effect reduce sensitivity. Sequential dissociation can be limited by reducing the irradiation time or laser intensity, but doing so also reduces parent ion fragmentation efficiency. The irradiation time necessary to produce as informative a tandem mass spectrum as possible with IRMPD must therefore be determined empirically [15]. The irradiation time also varies for different peptides and proteins with different IR activities and vibrational degrees of freedom. The trapping $\mathrm{rf}$ amplitude is a factor in determining the irradiation time because of the effect on ion cloud size; the rate of IR absorption is dependent on the amount of overlap between the ion cloud and the laser path $[10,16]$. The empirical determination of irradiation time for each different IRMPD experiment limits the real-time throughput with the technique. Continuous sequential dissociation from any irradiation time must compromise between product ion abundance and parent ion fragmentation efficiency.

The most effective way to limit continuous sequential dissociation without reducing parent ion fragmentation efficiency is to remove product ions from the laser path. One method of reducing sequential dissociation is by axial expansion of product ions [2]. A product ion is resonantly excited with a supplemental rf potential so that it has larger amplitude of axial motion in the trap. The axially expanded ion spends less time in the center of the trap where the laser beam is most intense. Sequential dissociation of the axially expanded product ion is reduced, although the ion cloud still overlaps and passes through the laser to some extent. Also, axial expansion can effect collision-induced dissociation for ions that fragment easily. Axial expansion is not completely selective because peptide product ions that have at least moderate IR activity can still dissociate under the reduced irradiation conditions. Simultaneous broadband excitation of product ions at different mass-to-charge and $\mathrm{q}_{\mathrm{z}}$ values causes differential axial expansion and may result in some losses of the ions intended for preservation. Any product ions not axially expanded continue to be sequentially dissociated in the laser path, reducing overall product ion abundance and MS/MS efficiency.

The work presented here describes a new IRMPD methodology in which sequential dissociation is controlled for all product ions. The laser beam has been translated axially away from the center of the trap to minimize the overlap with trapped ions under standard trapping conditions. A supplemental broadband $\mathrm{rf}$ voltage is applied to axially expand selected ions into the laser path where they are dissociated. Product ions are not axially expanded and kinetically cool to the center of the trap away from the laser, limiting the amount of sequential dissociation. This technique, termed selective broadband (SB) IRMPD, is shown to increase sensitivity by increasing the abundance of each product ion compared with conventional IRMPD at $3.3 \times 10^{-4}$ Torr. By making the IRMPD process selective, SB-IRMPD gains the advantages of selective CID and conventional IRMPD without the disadvantages of either, simultaneously maximizing fragmentation efficiency and MS/MS efficiency. Thermal assistance allows SB-IRMPD to be performed at bath gas pressures up to $5.2 \times 10^{-4}$ Torr, increasing product ion abundance to levels equal or greater than at thermally assisted (TA)-IRMPD at $1.0 \times 10^{-3}$ Torr.

\section{Experimental}

All experiments were performed on a customized Finnigan ITMS controlled with ICMS software [17]. Peptides and proteins were obtained from Sigma Chemical Co. (St. Louis, MO, USA) and used without further purification. Samples were prepared as $25 \mu \mathrm{M}$ solutions in 75:20:5 methanol: water:acetic acid. A nano-electrospray source was used to generate parent ions. Base pressure in the ion trap without added bath gas was $2 \times 10^{-5}$ Torr. Helium bath gas was added up to a total pressure of $3.3 \times 10^{-4}$ Torr for ambient temperature IRMPD experiments, the pressure just below where significant collisional cooling starts to occur [2, 12]. At greater than ambient temperatures helium bath gas was added up to a total pressure of $1 \times 10^{-3}$ Torr for IRMPD experiments. A Lesker $1000 \mathrm{~W}$ stab-in bakeout heater near the trapping electrodes is used to heat the bath gas and electrodes. The temperature is measured by a k-type thermocouple in the vacuum chamber located $2 \mathrm{~cm}$ from the trapping electrodes.

A Synrad $50 \mathrm{~W} \mathrm{CO}_{2}$ laser triggered by a TTL pulse from the ITMS electronics is used for IRMPD. The laser beam is passed into the vacuum housing through a $\mathrm{ZnSe}$ window by 
optical elements mounted on translation stages. The ring electrode has been modified by drilling a $3.2 \mathrm{~mm}$ hole through the center, perpendicular to the axial direction. The laser beam is passed through the hole and out the opposite side of the ring electrode into a beam dump. The laser is initially aligned as described previously [10], and the unfocused laser remains centered for conventional IRMPD. For SB-IRMPD, a ZnSe lens with a focal length of $38.1 \mathrm{~cm}$ focuses the laser. The lens and other optical elements are axially offset $1.50 \mathrm{~mm}$ to one side, with the focused laser beam away from the trapped ion cloud.

In SB-IRMPD experiments, an arbitrary waveform generator is used to apply rf-voltage waveforms for resonant excitation to the end-cap electrodes to axially expand the trajectories of selected ions. Stored waveform inverse Fourier transform (SWIFT) is implemented with LabVIEW to construct the waveforms [18]. The trapping rf amplitude is initially set so that the parent ion has a $\mathrm{q}_{\mathrm{z}}$ of 0.10 . A 50 $\mathrm{mV}_{p-p}$ SWIFT waveform is applied to a range of massto-charge values $\pm 1 \mathrm{Da}$ around parent ion for axial expansion. The parent ion is expanded into the axially offset laser path until $100 \%$ fragmentation efficiency is achieved (Figure 1), and the tandem mass spectrum is recorded.

Another stage of SB-IRMPD is performed on a segment of higher mass-to-charge product ions to generate lower mass-to-charge product ions. After SB-IRMPD of the parent ion, the trapping $\mathrm{rf}$ amplitude is adjusted to change the $\mathrm{q}_{\mathrm{z}}$ to 0.10 for the mass-to-charge $1 \mathrm{Da}$ less than the singlycharged parent ion $\left([\mathrm{M}+\mathrm{H}]^{+}-1\right)$. If the parent ion charge state is unknown, the trapping $\mathrm{rf}$ is instead changed for a $\mathrm{q}_{\mathrm{z}}$ of 0.10 for the largest observed product ion. A second broadband SWIFT waveform with $50 \mathrm{mV}_{p-p}$ average amplitude is applied to resonantly excite product ions that have $\mathrm{q}_{\mathrm{z}}$ values $0.10-0.13$. The $50 \mathrm{mV}_{p-p}$ average amplitude was determined to be the maximum voltage that could be used without resonantly ejecting the ions at these $\mathrm{q}_{\mathrm{z}}$ values [19]. The range of product ions that are axially expanded is a

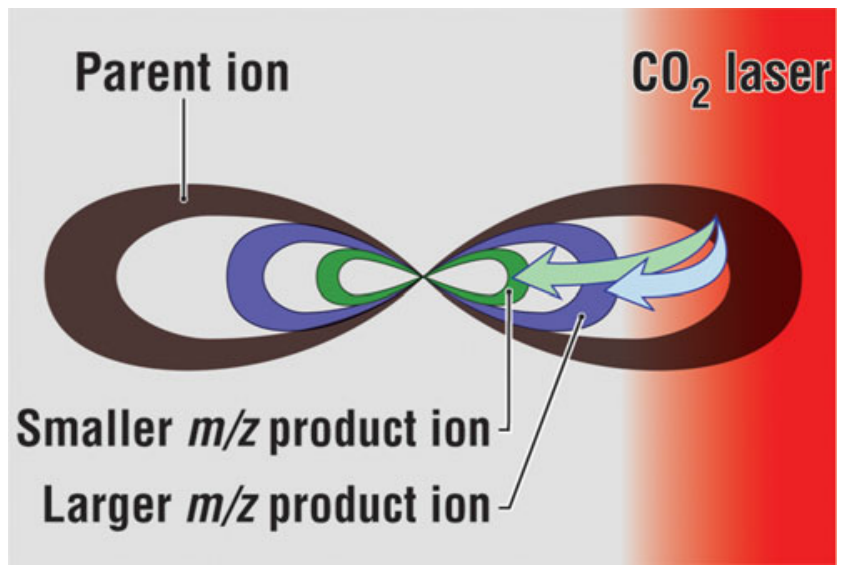

Figure 1. Graphical representation depicting the expansion of the parent ion cloud into the off-axis laser beam and the subsequent collisional cooling of the product ions out of the laser beam function of the mass-to-charge value. For example, if $\mathrm{m} / \mathrm{z}$ 600 is at a $\mathrm{q}_{\mathrm{z}}$ of 0.10 then $\mathrm{m} / \mathrm{z} 462$ is the lowest product ion in the range being excited. If $\mathrm{m} / \mathrm{z} 500$ is the product ion at $\mathrm{q}_{\mathrm{z}}$ of 0.10 then $\mathrm{m} / \mathrm{z} 385$ is the low end of the range. All excited product ions are axially expanded until completely dissociated, and the tandem mass spectrum is acquired after dissociation. The trapping rf amplitude can be repeatedly adjusted to place progressively smaller product ions at the same $(0.10-0.13) \mathrm{q}_{\mathrm{z}}$ range and the experiment repeated. Tandem mass spectra are collected after each dissociation stage. Larger $\mathrm{q}_{\mathrm{z}}$ values for ions were used to dissociate product ions below $m / z 391$ for reasons explained in results. Product ions from $m / z$ 390-301 are dissociated at $\mathrm{q}_{\mathrm{z}}$ values of $0.103-0.133$, and product ions from $\mathrm{m} / \mathrm{z} 300-201$ are dissociated at $\mathrm{q}_{\mathrm{z}}$ values of $0.120-0.179$. Multiple product ions below $\mathrm{m} / \mathrm{z} 200$ were not dissociated simultaneously, but individual product ions below $\mathrm{m} / \mathrm{z} 200$ are selectively dissociated at a $\mathrm{q}_{\mathrm{z}}$ of 0.20 .

\section{Results and Discussion}

\section{Ion Cloud Size Affects Irradiation Time and Selectivity}

Fragmentation efficiency and selectivity are both dependent on the amount of overlap between the ion cloud and laser. The centered laser has good overlap with the trapped ion cloud for conventional IRMPD and fragmentation efficiency of $97 \% \pm 3 \%$ was achieved for [bradykinin $+2 \mathrm{H}]^{2+}$ in $40 \mathrm{~ms}$. The overlap is poor between the axially expanded ion cloud and the axially offset laser because less than one half of the expanded ion cloud is irradiated. Laser flux on the irradiated area was increased by focusing the laser [10]. SB-IRMPD of $[\text { bradykinin }+2 \mathrm{H}]^{2+}$ required $80 \mathrm{~ms}$ irradiation with the axially offset laser to achieve a fragmentation efficiency of $98 \% \pm 2 \%$. No resonant ejection was observed when selected ions were axially expanded without irradiation.

Although the timescale for SB-IRMPD is longer than conventional IRMPD, product ions that are not axially expanded are not dissociated. Trapped ion clouds that are not axially expanded or have collisionally cooled from an expanded axial amplitude are referred to as "nonexpanded." Ion cloud size increases with decreasing $\mathrm{q}_{\mathrm{z}}$ [16], and nonexpanded ion clouds at low $\mathrm{q}_{\mathrm{z}}$ have been measured to radii $<1 \mathrm{~mm}$ [10]. The focused laser beam had a radius of $0.50 \mathrm{~mm}$, and thus overlap with the nonexpanded ion cloud was negligible when the laser center was axially offset $1.50 \mathrm{~mm}$. Nonexpanded parent and product ions at $\mathrm{q}_{\mathrm{z}}$ values as low as 0.03 exhibited $0 \%$ fragmentation efficiency after $200 \mathrm{~ms}$ irradiation with the axially offset laser.

Product ions formed during SB-IRMPD are not resonantly excited, but they initially have the same axial amplitude as the parent ion. A maximum of $20 \mathrm{~ms}$ of collisional cooling after dissociation is required for product ions to relax from an expanded axial amplitude, but most relax much faster than this $[12,20]$. During this period of 
collisional cooling, product ion clouds overlap with the laser for some period of time as opposed to a nonexpanded ion cloud, which has no overlap. Product ions also have increased internal energy because a portion of the photon energy absorbed by the parent ion is distributed to bonds that ultimately become part of product ions and thus, less additional IR absorption is required to dissociate the product ions compared with collisionally cooled ions. The amount of sequential dissociation depends on the IR activities and vibrational degrees of freedom of the product ions. SBIRMPD results in less sequential dissociation than conventional IRMPD and essentially no product ion formation below the LMCO. The amount of sequential dissociation is independent of the parent ion irradiation time once $100 \%$ fragmentation of the parent ion is achieved.

Product ions can be selectively dissociated with SBIRMPD in sequential stages to observe lower mass ions. To tune the parameters used for sequential dissociation, [GGYR $+\mathrm{H}]^{+}$was dissociated at different $\mathrm{q}_{\mathrm{z}}$ values (Figure 2). SBIRMPD fragmentation efficiency was reduced at $\mathrm{q}_{\mathrm{z}}$ values below 0.10 , but dissociation at larger $\mathrm{q}_{\mathrm{z}}$ values increases the probability that the smaller product ions formed will fall below the LMCO, reducing the MS/MS efficiency. The $\mathrm{q}_{\mathrm{z}}$ range $0.10-0.13$ was selected as the optimum for the tradeoff between fragmentation efficiency and MS/MS efficiency. Product ions below $\mathrm{m} / \mathrm{z} 391$ were dissociated in broadband segments at slightly larger $\mathrm{q}_{\mathrm{z}}$ values, as stated above, to take advantage of higher fragmentation efficiency without concern for ion loss because the LMCO is below the mass of immonium ions. While MS/MS efficiency should be less than or equal to the fragmentation efficiency it was sometimes measured to be greater than fragmentation efficiency due to the mass dependence of the electron multiplier, which detects lower mass ions more efficiently [21].

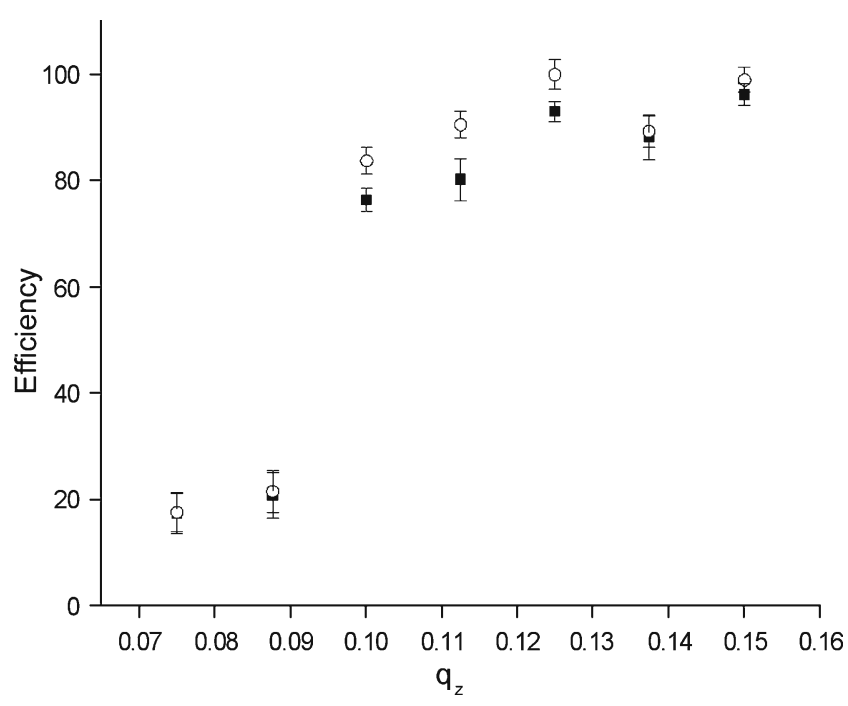

Figure 2. Fragmentation efficiency filled square ( $\bullet$ ) and MS/ MS efficiency open circle (०) observed from $50 \mathrm{~ms}$ SBIRMPD of $[\mathrm{GGYR}+\mathrm{H}]^{+}, 95 \%$ confidence error

\section{Conventional IRMPD Versus SB-IRMPD}

SB-IRMPD of parent ions gave greater absolute abundances of sequence ions and immonium ions, averaged across a single mass spectrum, than any single irradiation time in conventional IRMPD. The largest average product ion abundance from conventional IRMPD of [GRGDNP $+\mathrm{H}]^{+}$ was observed at $67 \% \pm 7 \%$ parent ion fragmentation efficiency (Figure 3a). When fragmentation efficiency was increased to $99 \% \pm 6 \%$ to observe more product ions, continuous sequential dissociation decreased the abundance of all product ions above $\mathrm{m} / \mathrm{z} 200$ (Figure 3b). Smaller mass product ions, below $m / z 200$, are immonium and related product ions, the later formed by side-chain fragmentation and $\mathrm{NH}_{3}$ and $\mathrm{H}_{2} \mathrm{O}$ losses from immonium product ions. Smaller mass ions are also subject to dissociation into product ions below the LMCO. SB-IRMPD of the parent ion achieved $98 \% \pm 2 \%$ fragmentation efficiency (Figure 3c). The limited sequential dissociation gave greater abundance for product ions above $\mathrm{m} / \mathrm{z} 200$, effectively increasing the sensitivity of MS/MS.

Sequential SB-IRMPD of product ions further increased low mass ion abundances compared to conventional

(a)

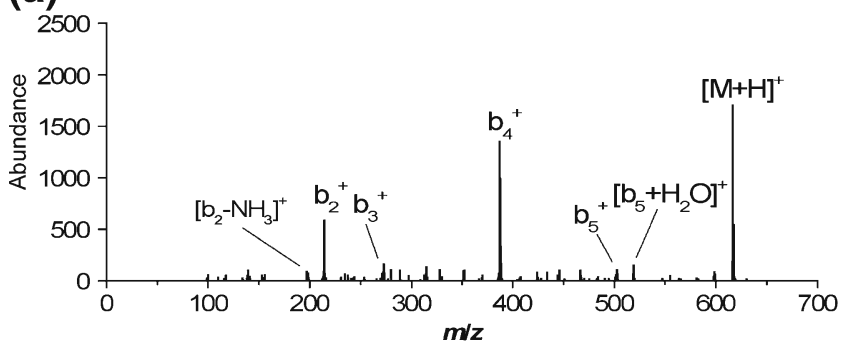

(b)
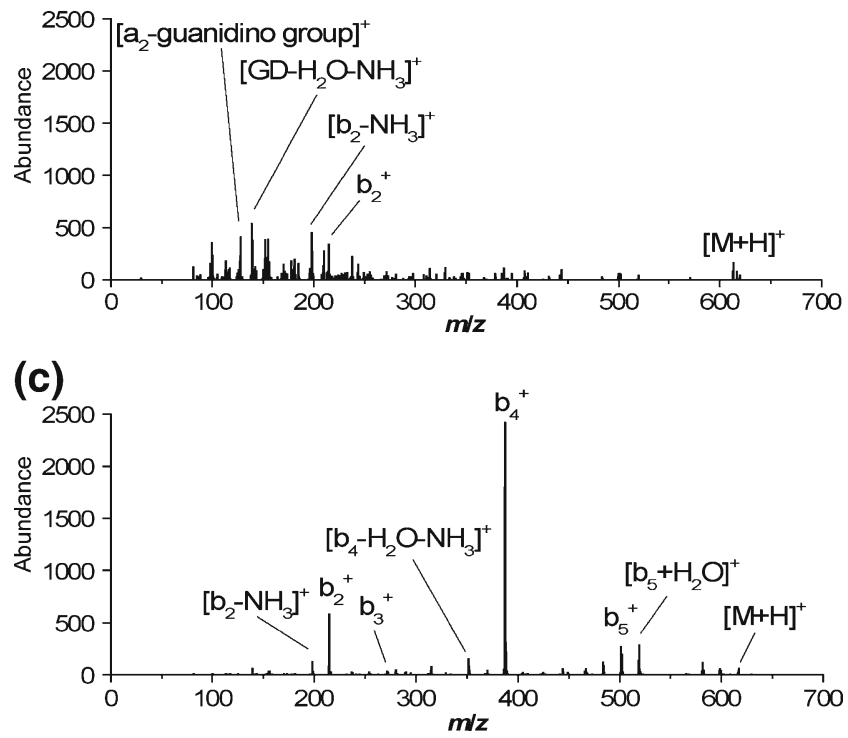

Figure 3. Tandem mass spectra of [GRGDNP $+\mathrm{H}]^{+}$from (a) conventional IRMPD, 67\% $\pm 7 \%$ fragmentation efficiency; (b) conventional IRMPD, $99 \% \pm 6 \%$ fragmentation efficiency; and (c) SB-IRMPD, 98\% $\pm 2 \%$ fragmentation efficiency 
IRMPD. Tables S1 and S2 (in Supplementary Material) list fragmentation efficiency values and product ion abundances from conventional IRMPD of [GRGDNP $+\mathrm{H}]^{+}$for comparison to sequential SB-IRMPD. For conventional IRMPD (Table S1) as fragmentation efficiency of the parent ion increased, product ion abundances grew to a maximum and then decreased due to continuous sequential dissociation. No single irradiation time gave the largest observed abundance for every product ion. Measured MS/MS efficiency was large at longer irradiation times because of detector bias toward low mass ions formed by continuous sequential dissociation, when most sequence ions and large product ions had low abundance. For SB-IRMPD the fragmentation efficiency could readily be set at $100 \%$. Product ion abundances from sequential stages of SB-IRMPD are listed in Table S2. The irradiation times used were the minimum necessary to achieve $100 \%$ dissociation of product ions in each broadband segment, and none required longer times than SB-IRMPD of the parent ion. Each successive dissociated broadband segment of ions increased the abundance for product ions at smaller mass-to-charge. For example, $\mathrm{b}_{4}{ }^{+}$at $\mathrm{m} / \mathrm{z} 386$ increased in abundance with sequential SB-IRMPD of the parent ion (broadband $\mathrm{m} / \mathrm{z}$ 614-616) and product ions (broadbands $m / z$ 471-614 and 391-471). SB-IRMPD of $\mathrm{b}_{4}{ }^{+}$in $m / z$ 301-390 then increased $\mathrm{b}_{3}{ }^{+}$and $\mathrm{b}_{2}{ }^{+}$abundance. Product ion abundances were $50 \%$ to $130 \%$ greater with sequential SB-IRMPD than the largest respective abundances observed at any irradiation time with conventional IRMPD (Table S1), with the exception of $\mathrm{b}_{3}{ }^{+}$ and the proline immonium ion $(\mathrm{P})$. The $\mathrm{P}$ immonium ion was only produced by sequential SB-IRMPD. MS/MS efficiency from SB-IRMPD of the parent ion was slightly smaller than conventional IRMPD because the limited sequential dissociation produced few ions below $\mathrm{m} / \mathrm{z} 200$, a best case scenario for conventional IRMPD. The high MS/ MS efficiency for SB-IRMPD is due to sequence related ions instead of low mass ions, which provide little sequence information from the high MS/MS efficiency experiment using conventional IRMPD. Measured MS/MS efficiency increased with sequential stages of SB-IRMPD for broadband ranges at higher mass, but then decreased some for lower mass broadband ranges because of formation of product ions below the LMCO.

Immonium ions and product ions small relative to parent ions have abundances that are more easily observed by SBIRMPD than conventional IRMPD. Immonium ions confirm the presence or absence of each residue in the parent ion [22]. In an extreme example of SB-IRMPD efficiency, the parent ion [ubiquitin $+10 \mathrm{H}]^{10+}$ and product ions at $\mathrm{m} / \mathrm{z} 201-$ 1200 were dissociated in eight sequential broadband segments to observe many low mass ions in high abundance (Figure 4). An immonium ion was observed for every residue in ubiquitin except $\mathrm{G}$. In addition, low mass ions related to R, Q, and $\mathrm{K}$ were observed at $\mathrm{m} / \mathrm{z} 84$ and 112; a low mass ion related to $\mathrm{N}$ was observed at $\mathrm{m} / \mathrm{z} 115$; and a low mass ion related to $\mathrm{H}$ was observed at $\mathrm{m} / \mathrm{z} 138$. Tworesidue internal fragment ions, many with $\mathrm{CO}, \mathrm{H}_{2} \mathrm{O}$, or $\mathrm{NH}_{3}$ losses, were also observed above $\mathrm{m} / \mathrm{z}$ 140. The great abundance of ions below $\mathrm{m} / \mathrm{z} 220$ and the mass dependence of the detector give a measured MS/MS efficiency of $640 \%$, i.e., approximately six singly charged low mass product ions are formed from each +10 charged parent ion.

Isomeric, $\mathrm{m} / \mathrm{z} 86 \mathrm{~L}$ and I immonium ions can be distinguished by dissociation products [23] and were observed in high abundance with sequential SB-IRMPD. Immonium ions from $\mathrm{L}$ and/or I residues were generated from sequential SB-IRMPD of $[\text { FLEEV }+\mathrm{H}]^{+}$and [FLEEI+ $\mathrm{H}]^{+}$. SB-IRMPD of $m / z 86$ from [FLEEV $\left.+\mathrm{H}\right]^{+}$produced $m / z$ 30 and 44 , but SB-IRMPD of $m / z 86$ from [FLEEI $+\mathrm{H}]^{+}$ caused $\mathrm{NH}_{3}$ loss from I for an additional product ion at $\mathrm{m} / \mathrm{z}$ 69 (Supplementary Material Figure S1).

\section{Thermally-Assisted SB-IRMPD}

Thermal assistance [2] allowed efficient SB-IRMPD to be performed at bath gas pressures above $3.3 \times 10^{-4}$ Torr for greater sensitivity. Raising the bath gas temperature decreases collisional cooling, and increases the initial internal energy of the parent ions thermal [24]. Smaller peptide ions have fewer IR-active modes and are more difficult to dissociate with IRMPD. SB-IRMPD of $452 \mathrm{Da}[\mathrm{GGYR}+\mathrm{H}]^{+}$ could not achieve $100 \%$ fragmentation efficiency in $150 \mathrm{~ms}$ at

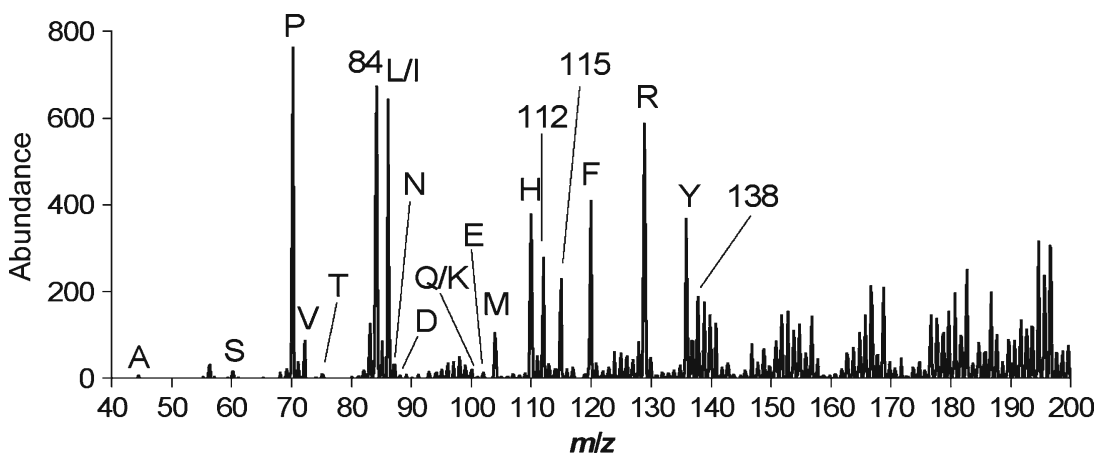

Figure 4. Tandem mass spectrum from sequential SB-IRMPD of [ubiquitin $+10 \mathrm{H}]^{10+}$, acquired after dissociating eight massto-charge broadband segments of ions in a total of $550 \mathrm{~ms}$. Immonium ions are labeled with one-letter symbols 
$3.3 \times 10^{-4}$ Torr and ambient temperature (Figure 5), and fragmentation efficiency was further decreased at higher pressures. Raising the bath gas temperature to $65^{\circ} \mathrm{C}$ or higher for thermally assisted (TA) SB-IRMPD increased fragmentation efficiency to $100 \%$ at $3.3 \times 10^{-4}$ Torr. TA-SB-IRMPD efficiency was also increased at higher bath gas pressures. Efficient TA-SB-IRMPD could not be performed at $1.0 \times$ $10^{-3}$ Torr because nonexpanded ions begin to dissociate at temperatures required for efficient IRMPD of the parent ion $\left(\geq 90^{\circ} \mathrm{C}\right)$.

Product ions from multiply-charged parent ions at a $\mathrm{q}_{\mathrm{z}}$ of 0.10 can have $\mathrm{q}_{\mathrm{z}}$ values near to or below the $\mathrm{q}_{\mathrm{z}}$ of the parent ion and have greater overlap with the axially offset laser than product ions at larger $\mathrm{q}_{\mathrm{z}}$. A larger peptide ion with many IRactive modes, $1424 \mathrm{Da}$ [granuliberin $\mathrm{R}+\mathrm{H}]^{2+}$, was used to model nonselective dissociation of nonexpanded product ion clouds at a $\mathrm{q}_{\mathrm{z}}$ of 0.033 (Figure 6). After $200 \mathrm{~ms}$ irradiation by the axially offset laser, significant dissociation was observed at $90^{\circ} \mathrm{C}$ for bath gas pressures above $4.6 \times 10^{-4}$ Torr. Thermal activation combined with a finite overlap and irradiation from the axially offset laser beam contributed to dissociation of the nonexpanded model ion cloud. Collisional cooling at high pressures and ambient temperature decreases fragmentation efficiency, but higher temperatures used to reduce collisional cooling can also reduce selectivity. A bath gas pressure of $5.2 \times 10^{-4}$ Torr and temperature of $85{ }^{\circ} \mathrm{C}$ gave the most efficient and sensitive TA-SB-IRMPD.

TA-SB-IRMPD at a bath gas pressure of $5.2 \times 10^{-4}$ Torr and temperature of $85^{\circ} \mathrm{C}$ gave greater product ion abundances than ambient temperature SB-IRMPD at $3.3 \times 10^{-4}$ Torr. The higher bath gas pressure for TA-SB-IRMPD increases trapping efficiency, and $[\mathrm{GRGDNP}+\mathrm{H}]^{+}$had $50 \%$ greater initial abundance before irradiation at $85^{\circ} \mathrm{C}$ and $5.2 \times 10^{-4}$ Torr than ambient temperature and $3.3 \times 10^{-4}$ Torr. Product ion abundances from TA-SB-IRMPD are shown in Table S3 (Supplementary Material) and were generally $15 \%-70 \%$ greater than

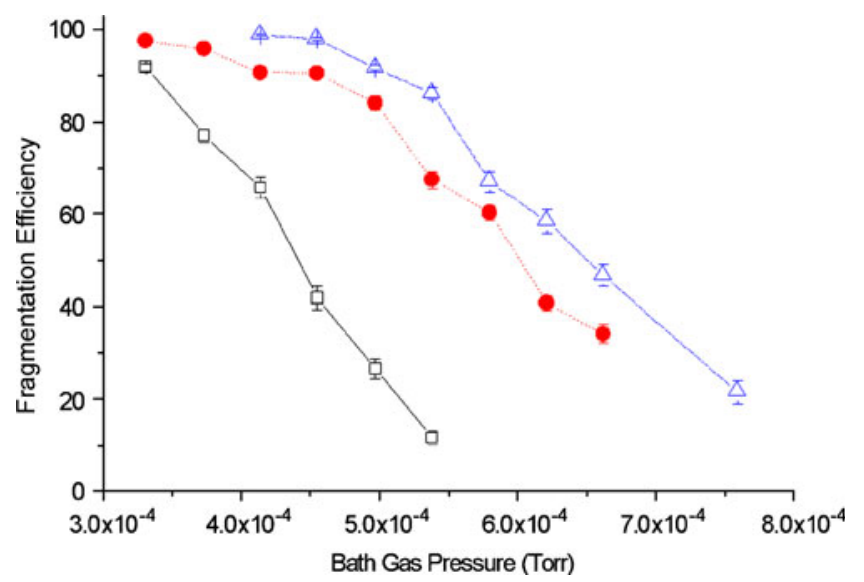

Figure 5. Fragmentation efficiency from $150 \mathrm{~ms}$ TA-SBIRMPD of [GGYR $+\mathrm{H}]^{+}$, at $a q_{z}$ value of 0.10 and a temperature of $30^{\circ} \mathrm{C}$ open square $(\square), 65^{\circ} \mathrm{C}$ filled circle $(\bullet)$, and $85^{\circ} \mathrm{C}$ open triangle $(\Delta)$ at various helium bath gas pressures, $95 \%$ confidence error

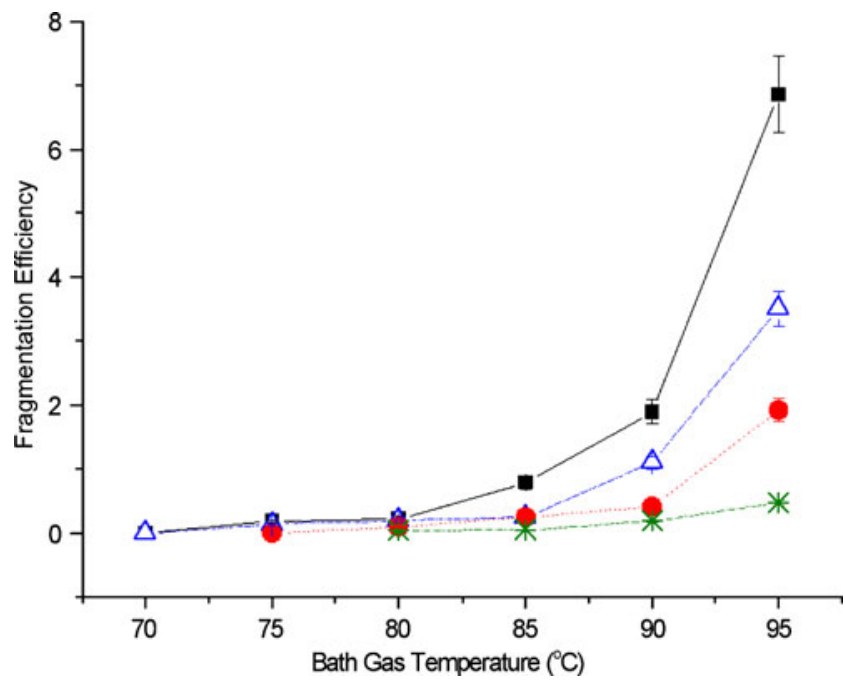

Figure 6. Fragmentation efficiency from $200 \mathrm{~ms}$ irradiation of [granuliberin $\mathrm{R}+\mathrm{H}]^{2+}$ nonexpanded ion cloud with axiallyoffset laser, at a $q_{z}$ value of 0.033 and a helium bath gas pressure of $4.6 \times 10^{-4}$ Torr filled square (घ), $5.2 \times 10^{-4}$ Torr open triangle $(\Delta), 5.9 \times 10^{-4}$ Torr filled circle $(\bullet)$, and $8.3 \times 10^{-}$ ${ }^{4} \operatorname{Torr}(X)$ at various temperatures, $95 \%$ confidence error

product ion abundances from ambient temperature SB-IRMPD in Table S2. Thermal activation increased sequential dissociation and decreased abundance of only the $\mathrm{b}_{4}{ }^{+}$ion. MS/MS efficiency was equal to measurements from ambient temperature SB-IRMPD, showing that sequential dissociation is limited about as effectively for TA-SB-IRMPD. The minimum irradiation time for completely dissociating ions in the same mass-to-charge broadband segment was reduced $40 \%$ with TA-SB-IRMPD.

TA-SB-IRMPD at $5.2 \times 10^{-4}$ Torr and $85{ }^{\circ} \mathrm{C}$ was also compared to TA-IRMPD at $1.0 \times 10^{-3}$ Torr and $150{ }^{\circ} \mathrm{C}$. The largest average product ion abundance from TA-IRMPD of $[\text { GRGDNP }+\mathrm{H}]^{+}$was observed at $76 \% \pm 4 \%$ parent ion fragmentation efficiency (Supplementary Material Table S4). As with conventional IRMPD, in TA-IRMPD continuous sequential dissociation caused product ion abundances to grow to a maximum and then decrease as parent ion fragmentation efficiency increased. The $1.0 \times 10^{-3}$ Torr bath gas gave optimal trapping efficiency, and the parent ion before irradiation had four times greater abundance than for TA-SB-IRMPD at 5.2× $10^{-4}$ Torr. However, TA-SB-IRMPD of the parent ion gave greater average product ion abundances than any single irradiation time for TA-IRMPD in Table S4. Half of all product ion abundances were greater with sequential stages of TA-SBIRMPD than the largest respective abundances observed at any irradiation time with TA-IRMPD. Sequential stages of TA-SBIRMPD produced the $\mathrm{P}$ immonium ion, as did ambient temperature SB-IRMPD.

\section{Conclusions}

SB-IRMPD combines the selectivity and ability to be automated of conventional CID with the small LMCO and 
ability to generate low mass ions of conventional IRMPD. Selectivity is achieved by axially offsetting the laser and resonantly exciting ions into the laser path. SB-IRMPD limits sequential dissociation and increases the abundance of product ions compared to conventional IRMPD. Sequence ions and low mass immonium and related ions are increased in abundance by sequential stages of SB-IRMPD. The maximum abundances of product ions from sequential SBIRMPD can be acquired without empirical determination of irradiation time, increasing research throughput by reducing method development. Thermal assistance is used to perform SB-IRMPD at bath gas pressures above $3.3 \times 10^{-4}$ Torr for even greater sensitivity. TA-SB-IRMPD increases product ion abundance over ambient temperature SB-IRMPD, and results are comparable to TA-IRMPD at $1.0 \times 10^{-3}$ Torr, the bath gas pressure for optimal sensitivity in a quadrupole ion trap. Overly high bath gas temperatures cause dissociation of ions that are not selected, and temperatures below $90{ }^{\circ} \mathrm{C}$ were not sufficient to make TA-SB-IRMPD efficient at $1.0 \times$ $10^{-3}$ Torr. The SB-IRMPD methodology could be further improved by increasing efficiency at $1.0 \times 10^{-3}$ Torr.

\section{References}

1. Stephenson, J.L., Booth, M.M., Shalosky, J.A., Eyler, J.R., Yost, R.A.: Infrared multiple photon dissociation in the quadrupole ion trap via a multipass optical arrangement. J. Am. Soc. Mass Spectrom.5, 886-893 (1994)

2. Payne, A.H., Glish, G.L.: Thermally assisted infrared multiphoton photodissociation in a quadrupole ion trap. Anal. Chem.73, 3542-3548 (2001)

3. Colorado, A., Shen, J.X., Vartanian, V.H., Brodbelt, J.: Use of infrared multiphoton photodissociation with SWIFT for electrospray ionization and laser desorption applications in a quadrupole ion trap mass spectrometer. Anal. Chem.68, 4033-4043 (1996)

4. Goolsby, B.J., Brodbelt, J.S.: Characterization of b-Lactams by photodissociation and collision-activated dissociation in a quadrupole ion trap. J. Mass Spectrom.33, 705-712 (1998)

5. Shen, J., Brodbelt, J.S.: Characterization of ionophore-metal complexes by infrared multiphoton photodissociation and collision-activated dissociation in a quadrupole ion trap mass spectrometer. Analyst125, 641-650 (2000)

6. Crowe, M.C., Brodbelt, J.S., Goolsby, B.J., Hergenrother, P.: Characterization of erythromycin analogs by collisional activated dissociation and infrared multiphoton dissociation in a quadrupole ion trap. J. Am. Soc. Mass Spectrom.13, 630-649 (2002)
7. Keller, K.M., Brodbelt, J.: Collisionally activated dissociation and infrared multiphoton dissociation of oligonucleotides in a quadrupole ion trap. Anal. Biochem.326, 200-210 (2004)

8. Yost, R.A., Enke, C.G.: Triple quadrupole mass-spectrometry for direct mixture analysis and structure elucidation. Anal. Chem.51, 1251-1264 (1979)

9. Louris, J.N., Brodbelt-Lustig, J.S., Cooks, R.G., Glish, G.L., Berkel, G. J.V., McLuckey, S.A.: Ion isolation and sequential stages of mass spectrometry in a quadrupole ion trap mass spectrometer. Int. J. Mass Spectrom. Ion Processes96, 117-137 (1990)

10. Newsome, G.A., Glish, G.L.: Improving IRMPD in a quadrupole ion trap. J. Am. Soc. Mass Spectrom.20, 1127-1131 (2009)

11. Stafford, G.C.J., Kelley, P.E., Syka, J.E.P., Reynolds, W.E., Todd, J.F. J.: Recent improvements in and analytical applications of advanced ion trap technology. Int. J. Mass Spectrom. Ion Processes60, 85-98 (1984)

12. Black, D.M., Payne, A.H., Glish, G.L.: Determination of cooling rates in a quadrupole ion trap. J. Am. Soc. Mass Spectrom.17, 932-938 (2006)

13. Hashimoto, Y., Hasegawa, H., Yoshinari, K., Waki, I.: Collisionactivated infrared multiphoton dissociation in a quadrupole ion trap mass spectrometer. Anal. Chem.75, 420-425 (2003)

14. Boué, S.M., Stephenson, J.L., Yost, R.A.: Pulsed helium introduction into a quadrupole ion trap for reduced collisional quenching during infrared multiphoton dissociation of electrosprayed ions. Rapid Commun. Mass Spectrom.14, 1391-1397 (2000)

15. Pikulski, M., Hargrove, A., Shabbir, S.H., Anslyn, E.V., Brodbelt, J.S.: Sequencing and characterization of oligosaccharides using infrared multiphoton dissociation and boronic acid derivatization in a quadrupole ion trap. J. Am. Soc. Mass Spectrom.18, 2094-2106 (2007)

16. Remes, P.M., Glish, G.L.: Mapping the distribution of ion positions as a function of quadrupole ion trap mass spectrometer operating parameters to optimize infrared multiphoton dissociation. J. Phys. Chem. A113, 3447-3454 (2009)

17. Yates, N.: Yost, R. ICMS Ion Trap Software, Release 2.20, (1992)

18. Asam, M.R., Ray, K.L., Glish, G.L.: Collision-induced signal enhancement: a method to increase product ion intensities in MS/MS and $\mathrm{MS}^{n}$ experiments. Anal. Chem.70, 1831-1837 (1998)

19. Charles, M.J., McLuckey, S.A., Glish, G.L.: Competition between resonance ejection and ion dissociation during resonant excitation in a quadrupole ion trap. J. Am. Soc. Mass Spectrom.5, 1031-1041 (1994)

20. Remes, P.M.: Instrumentation and methods for the characterization of ion structure and internal energy in the gas phase. Ph.D. Dissertation, University of North Carolina (2007)

21. Channeltron Electron Multiplier Handbook for Mass Spectrometry Applications. www.burle.com 2007

22. Papayannopoulos, I.A.: The interpretation of collision-induced dissociation tandem mass spectra of peptides. Mass Spectrom. Rev.14, 49-73 (1995)

23. Armirotti, A., Millo, E., Damonte, G.: How to discriminate between leucine and isoleucine by low energy ESI-TRAP MSn. J. Am. Soc. Mass Spectrom.18, 57-63 (2007)

24. Racine, A.H., Payne, A.H., Remes, P.M., Glish, G.L.: Thermally assisted collision-induced dissociation in a quadrupole ion trap mass spectrometer. Anal. Chem.78, 4609-4614 (2006) 